\title{
Strength of correlations in electron- and hole-doped cuprates
}

\section{Cédric Weber ${ }^{\star}$, Kristjan Haule and Gabriel Kotliar}

The introduction of holes in a parent compound consisting of copper oxide layers results in high-temperature superconductivity. It is also possible to dope the cuprate parent compound with electrons ${ }^{1-3}$. The physical properties of these electrondoped materials bear some similarities to but also significant differences from those of their hole-doped counterparts. Here, we use a recently developed first-principles method ${ }^{4}$ to study the electron-doped cuprates and elucidate the deep physical reasons behind their behaviour being so different from that of the hole-doped materials. The crystal structure of the electrondoped compounds is characterized by a lack of apical oxygens, and we find that it results in a parent compound that is a Slater insulator-a material in which the insulating behaviour is the result of the presence of magnetic long-range order. This is in sharp contrast with the hole-doped materials, which are insulating owing to the strong electronic correlations but not owing to magnetism.

The understanding of late-transition-metal oxides begins with seminal work by Zaanen et al. ${ }^{5}$, which established that the lowestenergy excitations in materials such as the copper oxides are the charge-transfer excitations of an electron from the oxygen anions to the copper cation. If the energy cost of the charge-transfer process is less then the kinetic energy gain resulting from this process, the system is metallic. Otherwise it is insulating. Materials on the metallic side of this metal-insulator boundary can turn into insulators if the band structure is such that Bragg scattering from the zone boundary can open a bandgap, as stressed in ref. 6. On the other hand, in the charge-transfer insulators, the magnetic order that occurs at lower temperatures is a consequence rather than the cause of the insulating behaviour.

The metal-insulator boundary is a 'mean-field' theoretical boundary separating the itinerant and the localized regimes of the low-energy electronic excitations. Early studies on simplified model Hamiltonians $^{7-10}$ substantiated this picture and showed that the actual copper oxides are not far from this metal-insulator boundary.

Here we go beyond model studies by incorporating realistic crystal structure and the interplay with magnetism. We study both the electron- and hole-doped cuprates. We find that the parent compound $\mathrm{NdCuO}_{4}$ of the electron-doped cuprate lies on the metallic side of the metal-insulator boundary. $\mathrm{NdCuO}_{4}$ is hence an insulator only as a result of the magnetic long-range order. This is in sharp contrast with the hole-doped cuprates, where the parent compound is a charge-transfer insulator.

We study the single-layer electron-doped compound $\mathrm{Nd}_{2-x} \mathrm{Ce}_{x} \mathrm{CuO}_{4}$ (NCCO), and we compare it with $\mathrm{La}_{2-x} \mathrm{Sr}_{x} \mathrm{CuO}_{4}$ (LSCO), a single-layer hole-doped material in the related $T$ structure. We use a realistic theoretical approach, the local-density approximation combined with the dynamical mean-field theory (LDA+DMFT; refs 4,11,12).
The theoretical results are expressed in terms of the electronic spectral functions $A(\omega)=-1 / \pi \operatorname{Im} G(\omega)$. The DMFT equations for NCOO have two solutions, shown in Fig. 1a,b. The first one is non-magnetic and metallic, and describes a material in the absence of long-range order. The second is insulating and antiferromagnetically ordered, with a charge-transfer gap of $1.2 \mathrm{eV}$. As the non-magnetic solution is metallic, the magnetic long-range order is responsible for the insulating nature of the compound.

Figure 1d zooms the low-energy part of panel b. We find that the low-energy states have mostly $d$ character with a significant oxygen $p$ admixture. In Fig. 1c we resolve the electronic states of panel $d$ in momentum space. We find two dispersive peaks separated by the charge-transfer gap. The top of the lower band occurs at $(\pi / 2, \pi / 2)$, and the bottom of the upper band appears at $M=(\pi, 0)$; therefore, the gap is indirect (see the yellow arrow in Fig. 1c). Those two bands can also be obtained in the simpler Hartree-Fock approximation, though the size of the gap is overestimated in a static mean field.

Figure 1e,f displays the correlation effects of the spectral function at $10 \%$ electron doping in the magnetic phase. The two-peak structure of the low-energy quasiparticles is clearly seen in the angle-integrated spectra of Fig. 1f. This comes primarily from the pseudogap around the momentum point $(\pi / 2, \pi / 2)$, which is a signature of magnetic long-range order (the Fermi surface of the ordered state is gapped at $(\pi / 2, \pi / 2)$, and moves towards the usual Fermi arc shape when the system becomes metallic).

The pseudogap is also observed in experiments ${ }^{13}$ (Fig. 1g, right) in the ordered phase, and compares well to our theoretical calculations (Fig. 1g, left). The pseudogap closes when the magnetic moment disappears.

In addition to the pseudogap around the $(\pi / 2, \pi / 2)$ point of the momentum-resolved spectra in Fig. 1e, there is an additional two-peak structure of the low-energy quasiparticles centred at the $\mathrm{M}$ point. We display the frequency dependence of these electronic states at a representative momentum point, $(3 \pi / 4,3 \pi / 4)$, in Fig. 1h. The peak positions are in a very good agreement with recent angle-resolved photo-emission measurements of ref. 13, also shown in Fig. 1h.

Some aspects of the doped electronic structure can be understood in terms of the Hartree-Fock rigid-band picture; for example, the holes appear first on doping at the $M=(\pi, 0)$ point, but the renormalization of the bands, and the multiple-peak structure in energy for a given momentum point (see Fig. 1h), are not captured in static mean field theory.

We now turn to the theoretical optical conductivity. We computed this quantity using the Kubo formula of linear response theory. It is shown in Fig. 2a, and is in good qualitative agreement with the experimental results ${ }^{14,15}$ reproduced in the right inset of Fig. 2a. The undoped compound has a sharp onset at an energy of the order of $1.5 \mathrm{eV}$, which we interpret as the direct gap, slightly 


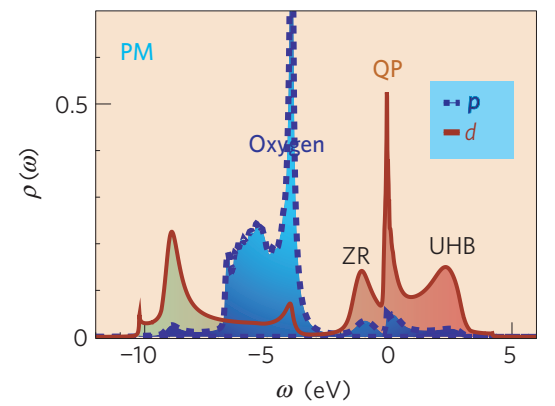

c

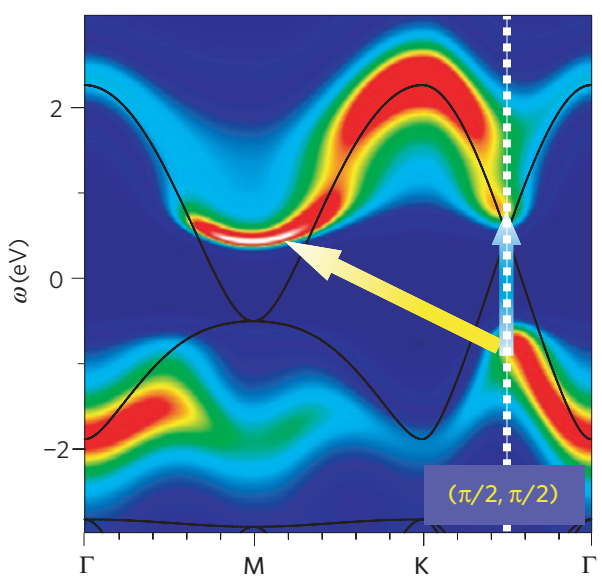

e

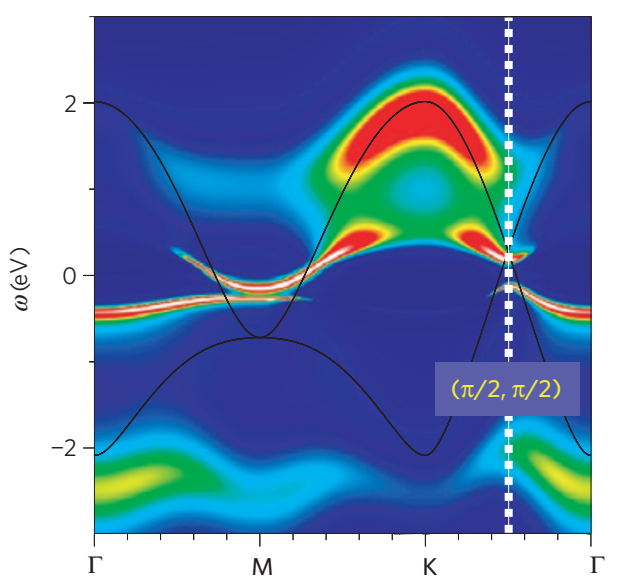

g
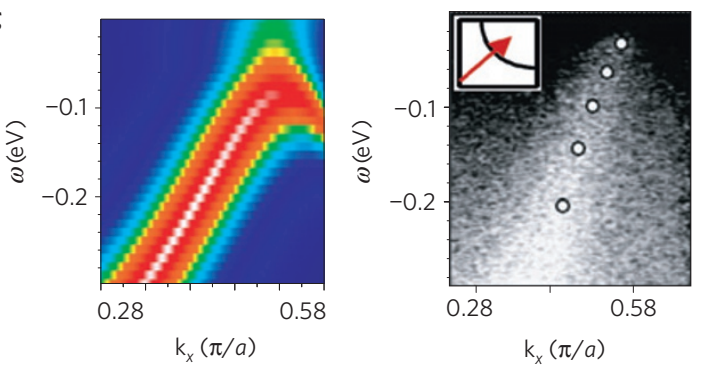

b

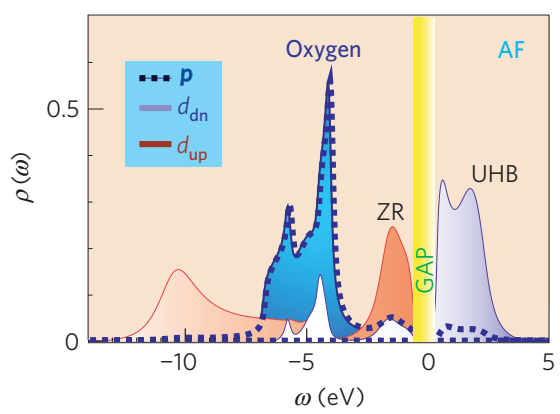

d

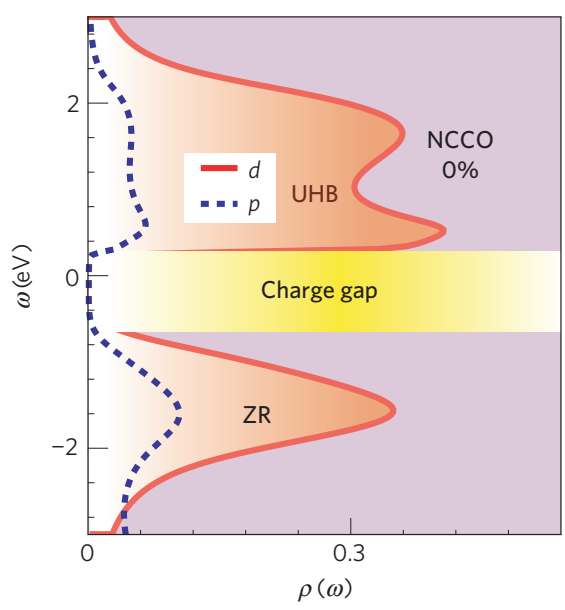

f

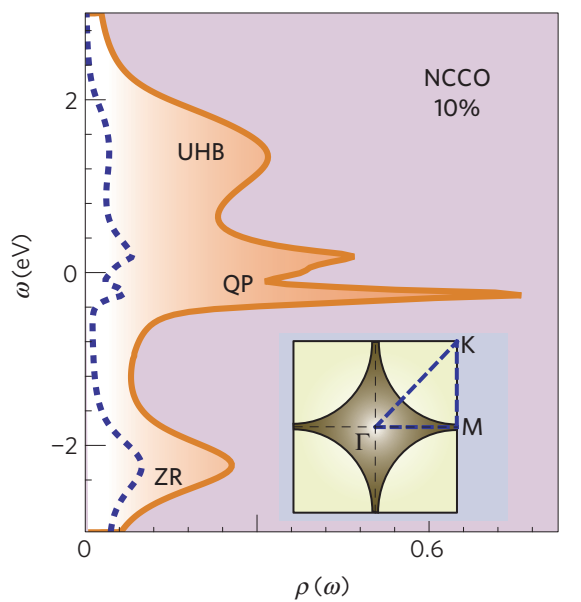

h

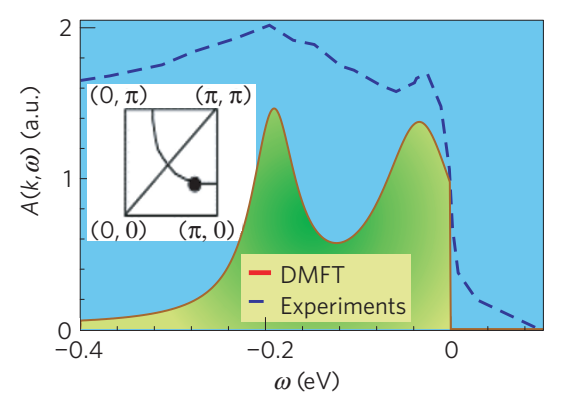

Figure 1 | Density of electronic states in NCCO. $\mathbf{a}, \mathbf{b}$, Spectral function of NCCO (a, non-magnetic; $\mathbf{b}$, magnetic) at zero doping in the extended energy range. There is a gap of $1.2 \mathrm{eV}$ when magnetism is allowed. We clearly observe the presence of the upper Hubbard bands (UHB), the Zhang-Rice singlet $(Z R)$ and a quasiparticle peak in the non-magnetic compound (QP). c,e, Frequency-dependent spectral weight $A(k, \omega)$ along $\Gamma-M-K-\Gamma$ obtained by LDA + DMFT at integer filling (c) and 10\% electron doping (NCCO) (e). d,f, The partial densities of states of the $d$ and $p$ orbitals. The solid lines in $\mathbf{c}$ and $\mathbf{e}$ are the LDA rigid bands, plotted in the folded Brillouin zone, for comparison with DMFT. The corresponding integrated weight is plotted in $\mathbf{d}$ and $\mathbf{f}$. $\mathbf{g}$, Side-by-side comparison of $A(k, \omega)$, along the path $\Gamma-K$ as depicted in the inset, obtained theoretically (left) and experimentally from ref. 13 (right) at $13 \%$ doping. $\mathbf{h}$, Comparison of $A(\omega)$ for a fixed $k$ point $k=(3 \pi / 4, \pi / 4)$ (shown in the inset) obtained theoretically (lower curve) and experimentally from ref. 13 (upper curve). Results in $\mathbf{b}-\mathbf{h}$ were obtained in the antiferromagnetic state. 


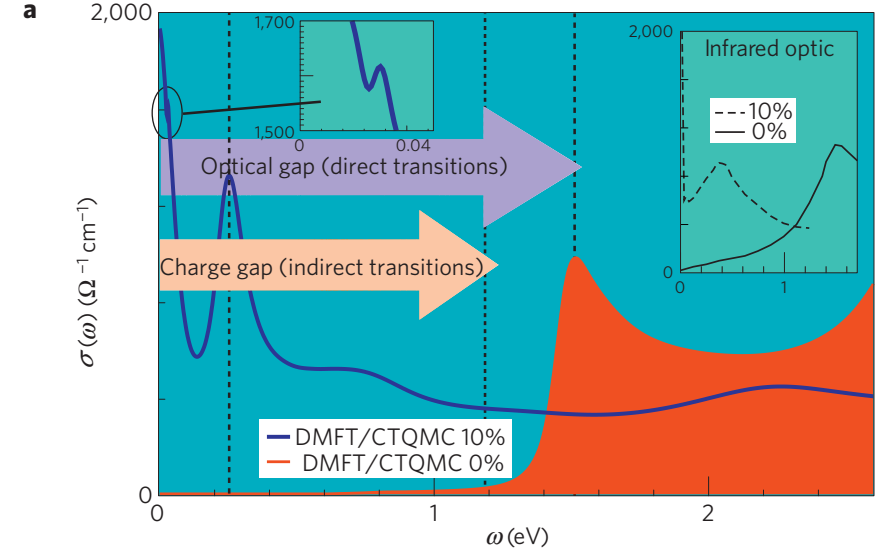

b

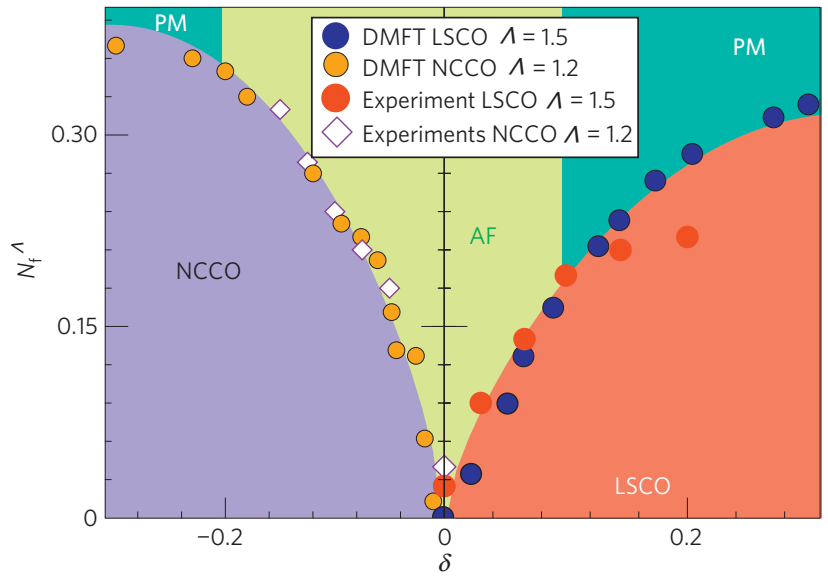

Figure 2 | Optical properties of NCCO. a, Theoretical optical conductivity in $\Omega^{-1} \mathrm{~cm}^{-1}$ for NCCO doped with charge carrier concentration at integer filling (red curve) and at $10 \%$ doping (blue curve). At integer filling, the system is gapped and we observe an optical gap of about $1.5 \mathrm{eV}$, which is larger than the charge gap, $\approx 1.2 \mathrm{eV}$. The left inset is a magnification of the data. For comparison we also show (right inset) the optical conductivity obtained by infrared optics ${ }^{14,15}$. b. The dimensionless integrated optical conductivity $N_{\text {eff }}$ for LDA+DMFT done on LSCO (ref. 12) and NCCO, obtained with a cutoff $\Lambda=1.2(\Lambda=1.5)$ for NCCO (LSCO). Experimental data for LSCO (ref. 19) (red circles) and NCCO (ref. 18) (open diamonds) are shown.

larger than the charge-transfer gap in Fig. 1d, and a tail resulting from indirect transitions.

Doping introduces several new features (blue line in Fig. 2a). The $1.5 \mathrm{eV}$ optical peak disappears and the weight is transferred to lower energy in the form of a Drude peak and a mid-infrared peak at $\omega \approx 0.2 \mathrm{eV}$. This mid-infrared peak has been identified as a spin-density-wave peak in ref. 16. In our spin-densitywave solution, it is connected to the multiple-peak structure of quasiparticles centred at the $M$ point in Fig. 1e, in particular to particle-hole excitations from the peak at $-0.2 \mathrm{eV}$ shown in Fig. $1 \mathrm{~h}$ and the unoccupied states.

Finally, the optical conductivity displays a peak in the magnetic solution at a much lower frequency $\omega \approx 0.035 \mathrm{eV}$ (see the left inset of Fig. 2a), which is connected to the pseudogap near $\mathbf{k}=(\pi / 2, \pi / 2)$ (see Fig. 1g). A similar feature, present only in the ordered state, is observed at this energy in experiments (see Fig. 2 of ref. 17). Our calculations show that this peak is connected to the pseudogap around $(\pi / 2, \pi / 2)$, present only in the ordered state.

To quantify the rate of redistribution of optical spectral weight, it is useful to consider the effective electron number per $\mathrm{Cu}$ atom defined by $N_{\text {eff }}=\left(2 m_{\mathrm{e}} V / \hbar \pi e^{2}\right) \int_{0}^{\Lambda} \sigma^{\prime}(\omega) \mathrm{d} \omega$, where $m_{\mathrm{e}}$ is the free-electron mass, $V$ is the cell volume containing one formula unit, $\Lambda$ is energy cutoff, and $\sigma^{\prime}$ is the real part of the optical conductivity. $N_{\text {eff }}$ is proportional to the number of electrons involved in the optical excitations up to the cutoff $\Lambda$. Our results for (electron-doped) NCCO are shown on the left-hand side of Fig. 2b and are compared with experimental data taken from ref. 18.

Notice a favourable agreement between theory and experiment, for which the use of the realistic electronic structure is essential.

In Fig. 2b (right) we also show our theoretical optical spectral weight for a prototypical hole-doped cuprate (LSCO), computed in ref. 12, which is a charge-transfer-type insulator, and we compare it with experimental data of ref. 19.

In a Slater picture, the onset of antiferromagnetism reduces the Coulomb correlations (double occupancy) at the expense of the kinetic energy. The opposite is true in a charge-transfer insulator. Consequently, in a Slater insulator the kinetic energy becomes less negative as the temperature decreases, whereas the opposite happens in a charge-transfer insulator. The kinetic energy as a function of temperature is readily available in the theory and is shown in Fig. 3a (top panel).

A closely related quantity $N_{\text {eff }}$ is both experimentally and theoretically accessible and has been measured for this compound. In Fig. 3b (top panel) we plot both the experimental ${ }^{18}$ and our theoretical data, which clearly follow the same trends. Hence the location of NCCO and LSCO, relative to the location of the metal-to-charge-transfer-insulator ${ }^{5}$ boundary, accounts for the observed trends in the temperature dependence of the optical conductivity. Similar trends of the temperature dependence of the kinetic energy for both electron- and hole-doped cuprates were reported in ref. 20.

In Fig. 3a (bottom panel) we also plot the temperature dependence of the kinetic energy and $N_{\text {eff }}$ for the hole-doped charge-transfer insulator (LSCO). We find the opposite trend compared with the lightly electron-doped NCCO compound, confirming that LSCO is a doped charge-transfer insulator, in agreement with experiments ${ }^{20}$.

When comparing with experiment, it is important to keep in mind that two-dimensional compounds are not able to sustain infinite-range magnetic order at a finite temperature (MerminWagner theorem ${ }^{21}$ ). Therefore, the Néel temperature within DMFT should be interpreted as the temperature below which the magnetic correlations become long-range but remain finite. This temperature can be much higher than the actual Néel temperature of the material, which is controlled by the magnetic exchange between the two-dimensional copper oxide layers, and vanishes for well separated copper oxide planes.

Finally, to check the validity of the single-site DMFT approach, we computed the magnetic-phase diagram and magnetic moment within single-site and two-site cluster DMFT (ref. 4) (see Fig. 3c). Our data for NCCO are shown on the left side of Fig. $3 \mathrm{c}$ and are compared with experimental data from ref. 22. The agreement is very good for both approaches, hence justifying the use of the single-site DMFT approach.

The right side of Fig. 3c displays theoretical and experimental magnetic moment ${ }^{23}$ for hole-doped LSCO. We found a significant difference in the region of stability of the magnetic state between the single-site and cluster DMFT $(\delta<10 \%)$, hence the dynamical short-range correlations-absent from the single-site approachare very important for LSCO in the underdoped regime, as reported in ref. 8, but not for NCCO.

We also carried out the Hartree-Fock calculation, and we found that in this static approach the magnetization vanishes only at unrealistically large doping $\delta \approx 50 \%$ for both NCCO and LSCO (dashed line in Fig. 3c), which points towards the important role of dynamic correlations at finite electron and hole doping. 
a

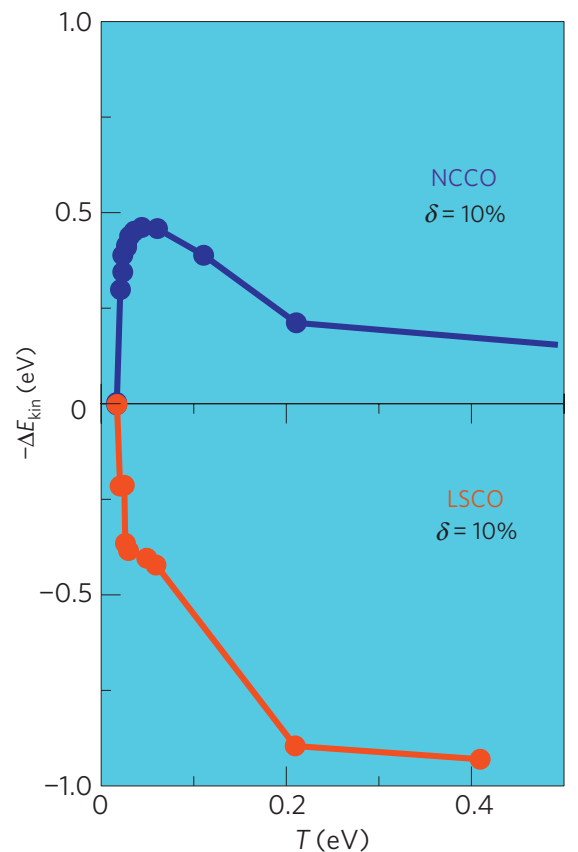

b

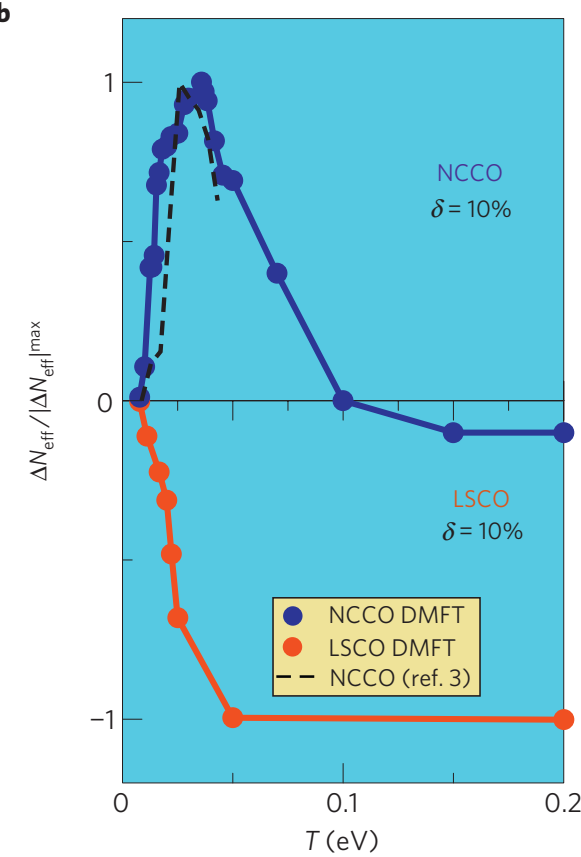

c

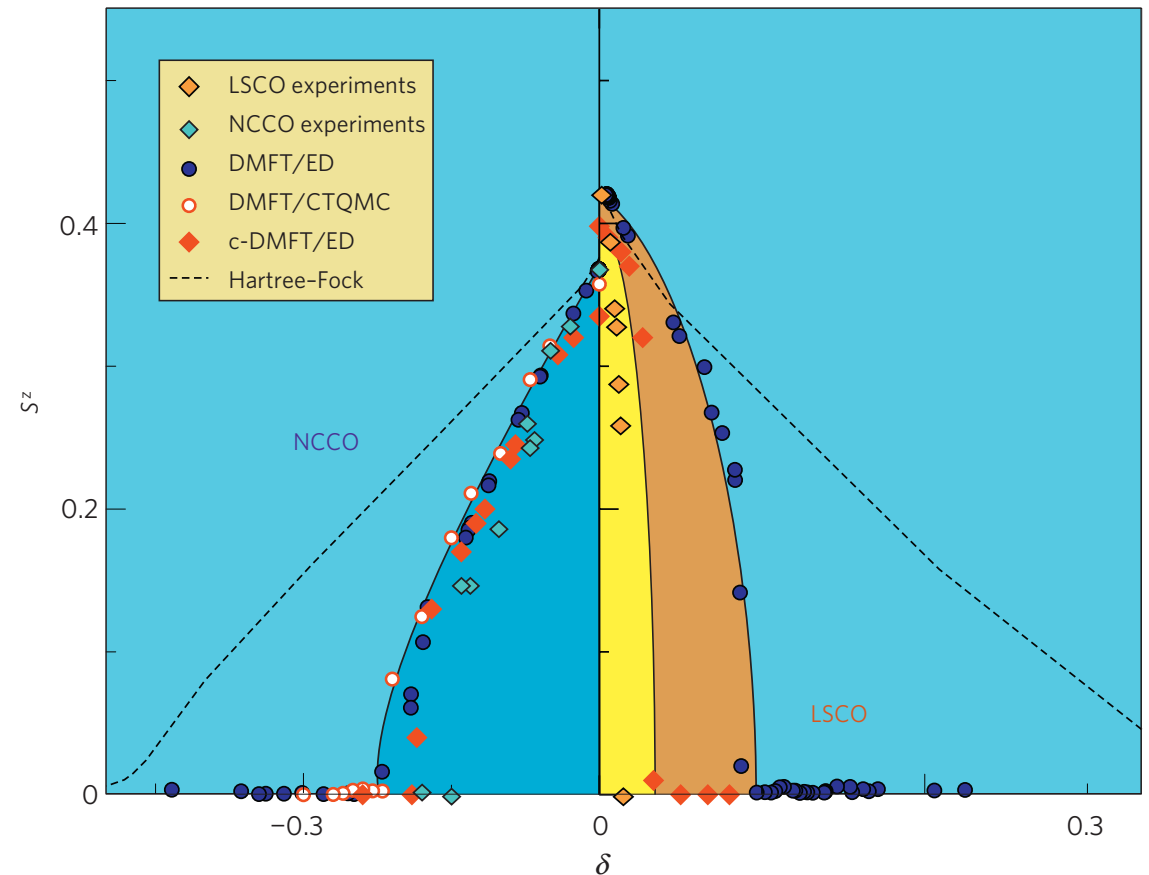

Figure 3 | Kinetic energy and magnetic-phase diagram. a, Theoretical kinetic-energy variation $-\Delta E_{\mathrm{kin}}=-E_{\mathrm{kin}}(T)+E_{\mathrm{kin}}(89 \mathrm{~K})$ of the electron- (top) and the hole- (bottom) doped compounds with respect to temperature. b, Normalized variation of $N_{\text {eff }}, \Delta N_{\text {eff }}=N_{\text {eff }}(T)-N_{\text {eff }}(89 \mathrm{~K})$ of the electron- (top) and the hole- (bottom) doped compounds with respect to temperature. The dashed line is extracted from experiments done on NCCO (ref. 18). c, Mean value of the staggered magnetization obtained for LSCO and NCCO by both single-site (DMFT) and cluster (c-DMFT) DMFT. Experimental values M( $\delta$ )/Mo $\left(M_{0}=M(\delta=0)\right)$ for NCCO (ref. 22) and LSCO (ref. 23) are also shown, and for comparison with DMFT we renormalized the experimental data by $M_{0}=M_{D M F T}(\delta=0)$. The magnetization obtained in the static Hartree-Fock approximation (dashed lines) is shown for comparison. Results obtained by exact diagonalization (DMFT/ED) and CTQMC (DMFT/CTQMC) are also shown.

Indeed, in the Hartree-Fock calculations, the picture is fairly simple: the magnetic state is stabilized owing to an optimization of the Coulomb energy at the expense of the kinetic energy (the staggered magnetic order avoids double occupation), and in this picture there is no strong difference between LSCO and NCCO, as shown in Fig. 3c.

Our theory sheds light on many puzzling observations. It has been noticed within the context of the one-band model that a doping-dependent Hubbard interaction was needed to reproduce experiments ${ }^{24}$. A realistic LDA+DMFT treatment of the electrondoped copper-oxygen system accounts for the rapid metallization process of the material, and hence does not require an ad hoc doping-dependent renormalization of the interaction.

Our main conclusion can be phrased succinctly in the language introduced in ref. 5: LSCO and NCCO lie on different sides of the metal/charge-transfer-insulator phase boundary. This provides 
a natural interpretation for the trends observed in the optical conductivity at finite doping as seen in Fig. 3b,c.

The link between the electronic properties and magnetism of the NCCO gives rise to additional experimental predictions. We expect that at high temperatures (of the order of the DFMT temperatures) there should be appreciable transfer of spectral weight from high energies to low energies with decreasing temperature, as the magnetic correlations are weakened, and a substantial decrease of the gap. This can be checked by extending transport and photoemission studies in lightly doped electron-doped cuprates from 600 to $1,100 \mathrm{~K}$.

The physical origin of the asymmetry between LSCO and NCCO lies not only in the different values of the oxygen-oxygen overlap, which controls the curvature of the Fermi surface, an effect that is captured in model Hamiltonian studies, but in the different values of the charge transfer gap in the two structures. The latter has an electrostatic origin: the electron-doped material lacks the negatively charged apical oxygen, which increases the electrostatic potential at the copper site.

In materials where the strength of the interaction is smaller than the critical value needed to produce a charge-transfer-insulating state, the effective interactions can renormalize, enabling superconductivity and magnetism to coexist microscopically rather than excluding each other. This coexistence provides a good description of the Raman scattering of the electron-doped superconductors ${ }^{25}$. Finally, in some NCCO films the parent compound was found to be metallic. It would be interesting to study the magnetic properties of these materials to see whether this metallicity correlates with a substantial decrease of the magnetic correlations ${ }^{26}$.

\section{Received 27 September 2009; accepted 14 May 2010;} published online 27 June 2010

\section{References}

1. Armitage, N. P. et al. Angle-resolved photoemission spectral function analysis of the electron-doped cuprate $\mathrm{Nd}_{1.85} \mathrm{Ce}_{0.15} \mathrm{CuO}_{4}$. Phys. Rev. B. 68, 064517 (2003).

2. Takagi, H. et al. Superconductivity produced by electron doping in $\mathrm{CuO}_{2}$-layered compounds. Phys. Rev. Lett. 62, 1197-1200 (1989).

3. Tokura, Y. et al. Electron and hole doping in Nd-based cuprates with single-layer $\mathrm{CuO}_{2}$ sheets: Role of doped Ce ions and 30-K superconductivity. Phys. Rev. B 39, 9704-9707 (1989).

4. Kotliar, G. et al. Electronic structure calculations with dynamical mean-field theory. Rev. Mod. Phys. 78, 865-951 (2006).

5. Zaanen, J. et al. Band gaps and electronic structure of transition-metal compounds. Phys. Rev. Lett. 55, 418-421 (1985).

6. Slater, J. C. Quantum Theory of Molecules and Solids Vol. 4. (McGraw-Hill, 1974).

7. Kotliar, G. Strong correlation transport and coherence. Int. J. Mod. Phys. B 5, 341-352 (1991).

8. Schmalian, J. et al. Doping dependence of local magnetic moments and antiferromagnetism in high-Tc superconductors: Asymmetry between electron and hole doping. Solid State Commun. 86, 119-122 (1993).
9. Baumgartel, G. et al. Theory for the electronic structure of high-Tc superconductors. Phys. Rev. B 48, 3983-3992 (1993).

10. Comanac, A. et al. Optical conductivity and the correlation strength of high temperature copper-oxide superconductors. Nature Phys. 4, 287-290 (2008).

11. Georges, A. et al. Dynamical mean-field theory of strongly correlated fermion systems and the limit of infinite dimensions. Rev. Mod. Phys. 68, 13-125 (1996).

12. Weber, C. et al. Optical weights and waterfalls in doped charge-transfer insulators: A local density approximation and dynamical mean-field theory study of $\mathrm{La}_{2-x} \mathrm{Sr}_{x} \mathrm{CuO}_{4}$. Phys. Rev. B. 78, 134519 (2008).

13. Matsui, H. et al. ARPES study of quasiparticle state in electron-doped cuprate $\mathrm{Nd}_{2} \mathrm{Ce}_{x} \mathrm{CuO}_{4}$. J. Phys. Chem. Solids 67, 249-253 (2006).

14. Xiang, T. et al. Intrinsic electron and hole bands in electron-doped cuprate superconductors. Phys. Rev. B 79, 014524 (2009).

15. Xiang, T. et al. Intrinsic electron and hole bands in electron-doped cuprate superconductors. Phys. Rev. B 79, 014524 (2009).

16. Zimmers, A. et al. Infrared properties of electron-doped cuprates: Tracking normal-state gaps and quantum critical behavior in $\operatorname{Pr}_{2-x} \mathrm{Ce}_{x} \mathrm{CuO}_{4}$ Europhys. Lett. 70, 225-231 (2005).

17. Onose, Y. et al. Doping dependence of pseudogap and related charge dynamics in $\mathrm{Nd}_{2-x} \mathrm{Ce}_{x} \mathrm{CuO}_{4}$. Phys. Rev. Lett. 87, 217001 (2001).

18. Onose, Y. et al. Charge dynamics in underdoped $\mathrm{Nd}_{2-x} \mathrm{Ce}_{x} \mathrm{CuO}_{4}$ : Pseudogap and related phenomena. Phys. Rev. B 69, 024504 (2004).

19. Uchida, S. et al. Optical spectra of $\mathrm{La}_{2-x} \mathrm{Sr}_{x} \mathrm{CuO}_{4}$ : Effect of carrier doping on the electronic structure of the $\mathrm{CuO}_{2}$ plane. Phys. Rev. B 43, 7942-7954 (1991).

20. Bontemps, N. et al. Temperature dependence of the spectral weight in p- and n-type cuprates: A study of normal state partial gaps and electronic kinetic energy. Ann. Phys. 321, 1547-1558 (2006).

21. Mermin, N. D. et al. Absence of ferromagnetism or antiferromagnetism in one- or two-dimensional isotropic heisenberg models. Phys. Rev. Lett. 17, 1133-1136 (1966).

22. Mang, P. K. et al. Spin correlations and magnetic order in non-superconducting $\mathrm{Nd}_{2-x} \mathrm{Ce}_{x} \mathrm{CuO}_{4 \pm \delta}$. Phys. Rev. Lett. 93, 027002 (2004).

23. Borsa, F. et al. Staggered magnetization in $\mathrm{La}_{2-x} \mathrm{Sr}_{x} \mathrm{CuO}_{4}$ from ${ }^{139} \mathrm{La}$ NQR and $\mu$ SR: Effects of Sr doping in the range $0<x<0.02$. Phys. Rev. B. 52, 7334-7345 (1995).

24. Kyung, B. et al. Pseudogap and spin fluctuations in the normal state of the electron-doped cuprates. Phys. Rev. Lett. 93, 147004 (2004).

25. Stadlober, B. et al. Is $\mathrm{Nd}_{2-x} \mathrm{Ce}_{x} \mathrm{CuO}_{4}$ a high-temperature superconductor? Phys. Rev. Lett. 74, 4911-4919 (1995).

26. Ismer, J-P. et al. Multiband superconductivity in spin density wave metals. Preprint at http://arxiv.org/abs/0907.1296 (2009).

\section{Acknowledgements}

We thank A. M. Tremblay, D. Basov, D. G. Hawthorn and G. A. Sawatzky for discussions and sharing their insights and experimental data. Discussions with A. Georges,

A. Amaricci, J. C. Domenge and A. Millis are acknowledged. J-C. Dommenge shared his exact diagonalization code. K.H was supported by Grant NSF NFS DMR-0746395 and the Alfred P. Sloan foundation. G.K. was supported by NSF DMR-0906943, and C.W. was supported by the Swiss National Science Foundation (SNSF).

\section{Author contributions}

C.W., K.H. and G.K. conceived the research; C.W. carried out numerical calculations; C.W. and K.H. contributed theoretical numerical codes; C.W. analysed the data; C.W., K.H. and G.K. wrote the paper; G.K. supervised the whole project.

\section{Additional information}

The authors declare no competing financial interests. Reprints and permissions information is available online at http://npg.nature.com/reprintsandpermissions. Correspondence and requests for materials should be addressed to C.W. 